\title{
Analysis and Detection Method for Machining Error of Globoidal Indexing Cam Profile
}

\author{
Sun Shuwen ${ }^{1}$, Jiang Zhengquan ${ }^{1}$, Huang $\mathrm{Jie}^{1}$, Yang Jianwu ${ }^{1}$
}

${ }^{1}$ No. 100, Pingleyuan, chaoyang district, Beijing, China

\begin{abstract}
The profile of globoidal indexing cam is spatial undevelopable surface. It needs special CNC machine tool to finish batch production. The machining quality of the profile of globoidal indexing cam could be affected by the movement error of each part of machine tool and the clamped positioning error of workpiece. Firstly, this paper analyses the systematic error of the special machine tool of globoidal cam, derives the error transfer matrix, establishes the mathematical model of the globoidal cam profile which including all machining system errors through multi-body system theory primarily. Secondly,this method improves the measuring speed and avoids the probe radius compensation, and evaluates the tolerance of the profile greed of globoidal cam equidistant model directly. Finally,the feasibility of the measurement method is verified by experiments, and the experimental results are analysed and processed. It lays the foundation for the design, process and measurement of globoidal cam mechanism and provides a theoretical basis and key technology for developing the online rapid detection system of the globoidal indexing cam profile.
\end{abstract}

\section{Introduction}

The globoidal cam indexing mechanism has the advantages of compact structure, high indexing precision, strong bearing capacity, good high-speed performance and so on, so it has been widely used in ATC of CNC machine tools, tobacco machinery, packaging machinery. Globoidal indexing cam is a key component in Automatic Tool changer (ATC) [1-3], because ATC requires high precision of globoidal cam profile, the manufacture and tolerance detection of high-grade globoidal cam has been a difficult problem for domestic enterprises. Therefore, the profile accuracy of the globoidal indexing cam must be strictly guaranteed in the manufacturing process. It is of great practical significance to research the machining error anddetection of globoidal cam [4].

There are a number of scholars who have studied the machining error of globoidal cam. Wang [5] from National Chiao Tung University analyzes the influence on output accuracy of globoidal indexing cam mechanism caused by the installation error of three moving directions and three rotating directions in space. H. Y. Cheng [6] from Da-Ye University analyzes the error transfer of globoidal indexing cam mechanism systematically, establishing the error transfer equation, at the same time, he analyzes the sensitivity made by output precision to each error, and establishing the sensitivity equation. Yin Mingfu [7] from Tianjin University of Technology researches on the influence caused by center distance error of globoidal indexing cam machine tool on cam profile error and gives the changing trend of error. Ji Shuting [8] [9] from Beijing University of Technology researches on the influence caused by center distance error of the globoidal indexing cam special machining on the cam profile machining error and draws the influence coefficient curve, she reveals the result that the center distance error leads to the error of globoidal indexing cam profile. On the basis of the above research, the influence caused by two rotation shaft errors, three vertical errors and three linear displacement errors of globoidal indexing cam special machining on the machining error of globoidal indexing cam profile have been analyzed [10]. Wang Haiyan puts forward two kinds of scanning measuring method [11] for measuring the globoidal cam by applying of CMM. Tang Lin measures in the CMM and introduced the concept [12] of profile (surface) tolerance and helix tolerance. P.D.Lin and J.F.Hsieh researched on the conversion between the measurement coordinates and the theoretical position, to determine the measuring benchmark [13]. Led by the Jilin Industrial University, the precision index system [14] of globoidal cam mechanism is put forward. Song Lijuan puts forward the thought that reversely getting the manufacturing tolerance by measuring results, but she doesn't research on the specific contents [15]. Lin Xiaojun proposed the method to compensate [16] the radius by reconstructing the equidistant surface. However, the measuring speed of current CMM method is slow and complex, it cannot meet the measurement requirements of globoidal cam in production line, and it is difficult to fundamentally solve the problem of radius compensation.

In this paper, based on the working principle of the globoidal cam machine, the mathematical model of the error of globoidal cam profile is established by using the multi-body system theory. A fast measuring method based 
on the equidistant model characteristic line of globoidal cam is proposed. This method can be used in the measurement of production line, and the measuring speed is fast, so the radius compensation is avoided effectively.

\section{Analysis of Cam Special Machine toolError}

\subsection{Processing Principle of Globoidal Cam}

The whole structure of special CNC machine tool for globoidal indexing cam is shown in Fig.1a, it uses four axes linkage pattern and generating method to process. The four axes are represented by " $A$ ", "B", "W" and "Z" respectively. Axis $A$ and axis $B$ are rotating axes, axis $W$ and axis $Z$ are linear moving axes. While machining globoidal indexing cam, it should not only rotates around its own axis but also swing. The two motions are realized and controlled by the motion of the mutually interactive axis $A$ and $B$ respectively; the distance between axis $A$ and axis $B$ is the center distance of globoidal cam indexing mechanism which is adjusted and controlled by axis $W$; axis Zrealizes the linear feed motion of control cutter along its central axis. The relative motion between workpiece and cutter realized by the linkage of axis $A$ and axis $B$ is equivalent to the pre-designed relative motion between globoidal indexing cam and driven turntable in the globoidal cam mechanism.

\subsection{Definition of Cam Special Machine Tool Error}

It is necessary to consider the many kinds of machine tool errors in the manufacturing process of the globoidal indexing cam. According to the multi-body theory, the motion error model is shown in Fig.1. At this time, there are two branches in the machine tool processing system.

Cutter branch: machine tool body----spindle sliding table axisZ----spindle----cutter;

Cam branch: machine tool body----turntable axis $B$----powered support axis $W$----rotary axis $A$----globoidal cam.

There are three linear displacement errors and three angular displacement errors in the spindle, axis $A$, axis $B$, axis $Z$ and axis $W$. And it is also necessary to consider the error of cam rotation angle and driven turntable rotation angle and the centre distance error between camshaft and driven shaft, since there is relative motion relation between the cam rotation angle and the driven turntable rotation angle. In order to facilitate understanding, describing the above error decomposition diagram according to the machine tool structure in Fig.1, as shown in Fig.2. The error definition of globoidal cam machine too and symbolic representation are shown in table 1.

Fig. 2 defines the error coordinates of moving parts of the globoidal indexing cam special machine tool. Fig.2ais themachine tool bed, Fig. $2 \mathrm{~b}$ is axis $B$, Fig. $2 \mathrm{c}$ is axis $W$, Fig.2d is axis $A$, Fig.2e isthe spindle,Fig. $2 \mathrm{f}$ is axis $Z$.
The feature transformation matrix of multi-body system is represented by homogeneous coordinate. Homogeneous coordinate transformation includes six transformation modes: linear displacement along three coordinate axes and angular displacement around three coordinate axes. According to the theory of homogeneous coordinate transformation, we can get each feature transformation matrix between each moving body and its adjacent low-sequence body. Therefore, the transformation matrix of error coordinate system of spindle error of machine tool, the axis B, rotating axis $\mathrm{A}$, power support axis $\mathrm{W}$ and the spindle sliding table axis $\mathrm{Z}$ can be obtained. The transformation matrix includes linear displacement matrix and angular Displacement matrix.

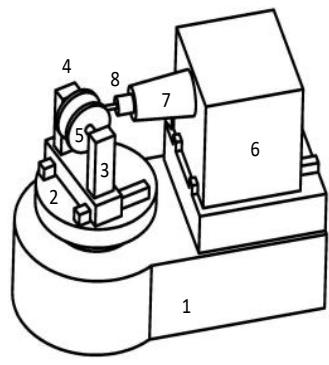

a) machine structure

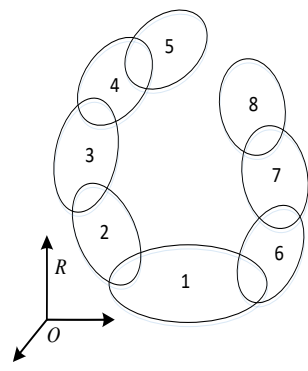

b) topological graph
1 machine tool bed

$2 B$-axial

$3 W$-axial

4 A-axial

5 globoidal cam

6 Z-axial

7 main shaft

8 tool

Figure1. Topology diagram of special machine tool

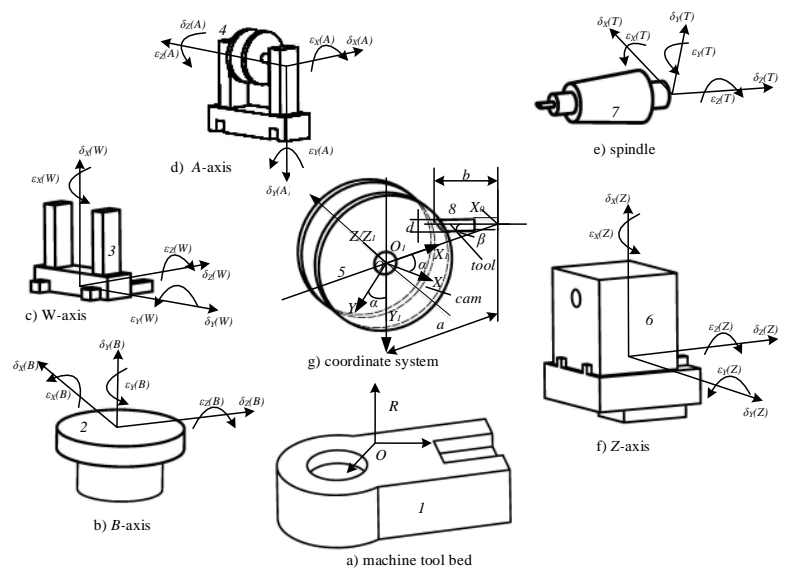

Figure2. Error definition of special machine tool

Taking the transformation matrix of the error coordinate system about machine tool spindle as an example, the linear displacement transformation matrix includes: the $t_{T X}$ of $X$ axis direction, the $t_{T Y}$ of $Y$ axis direction, the $t_{T Z}$ of axis $Z$ direction which are the error coordinate system along machine tool spindle. The transformation matrix of angular displacement includes: error coordinate system around machine tool spindle----the $r_{R X}$ which rotates around $X$ axis, the $r_{R Y}$ which rotates around the $Y$ axis, the $r_{R Z}$ which rotates the axis $Z$.

$$
t_{T X}=\left[\begin{array}{cccc}
1 & 0 & 0 & \delta_{X}(T) \\
0 & 1 & 0 & 0 \\
0 & 0 & 1 & 0 \\
0 & 0 & 0 & 1
\end{array}\right]
$$

\subsection{Feature Transformation Matrix.}




$$
\begin{aligned}
t_{T Y} & =\left[\begin{array}{llll}
1 & 0 & 0 & 0 \\
0 & 1 & 0 & \delta_{Y}(T) \\
0 & 0 & 1 & 0 \\
0 & 0 & 0 & 1
\end{array}\right] \quad(2) \\
t_{T Z} & =\left[\begin{array}{llll}
1 & 0 & 0 & 0 \\
0 & 1 & 0 & 0 \\
0 & 0 & 1 & \delta_{Z}(T) \\
0 & 0 & 0 & 1
\end{array}\right] \\
r_{R X} & =\left[\begin{array}{ccccc}
1 & 0 & 0 \\
0 & \cos \left(\varepsilon_{X}(T)\right) & \sin \left(\varepsilon_{X}(T)\right) & 0 \\
0 & -\sin \left(\varepsilon_{X}(T)\right) & \cos \left(\varepsilon_{X}(T)\right) & 0 \\
0 & 0 & 0 & 1
\end{array}\right] \\
r_{R Y} & =\left[\begin{array}{cccc}
\cos \left(\varepsilon_{Y}(T)\right) & 0 & -\sin \left(\varepsilon_{Y}(T)\right) & 0 \\
0 & 1 & 0 & 0 \\
\sin \left(\varepsilon_{Y}(T)\right) & 0 & \cos \left(\varepsilon_{Y}(T)\right) & 0 \\
0 & 0 & 0 & 1
\end{array}\right] \\
r_{R Z} & =\left[\begin{array}{cccc}
\cos \left(\varepsilon_{Z}(T)\right) & \sin \left(\varepsilon_{Z}(T)\right) & 0 & 0 \\
-\sin \left(\varepsilon_{Z}(T)\right) & \cos \left(\varepsilon_{Z}(T)\right) & 0 & 0 \\
0 & 0 & 1 & 0 \\
0 & 0 & 0 & 1
\end{array}\right]
\end{aligned}
$$

Similarly, the linear displacement and angular displacement matrix of error coordinate system of turntable axis $B$, the rotating axis $A$, the power support axis $W$ and spindle slide table of $Z$ can be obtained respectively. The representation method of the transformation matrix of each axis about the machine tool are shown in table 2 .

Table1. Errors of the special machine tool

\begin{tabular}{lcccccc}
\hline \multicolumn{1}{c}{ Error Type } & \multicolumn{3}{c}{ Linear offset error } & \multicolumn{3}{c}{ Rotational error } \\
\hline Error & $X$ & $Y$ & $Z$ & $X$ & $Y$ & $Z$ \\
Direction & & & & & \\
Spindle & $\delta_{X}(T)$ & $\delta_{Y}(T)$ & $\delta_{Z}(T)$ & $\varepsilon_{X}(T)$ & $\varepsilon_{Y}(T)$ & $\varepsilon_{Z}(T)$ \\
Z-axis & $\delta_{X}(Z)$ & $\delta_{Y}(Z)$ & $\delta_{Z}(Z)$ & $\varepsilon_{X}(Z)$ & $\varepsilon_{Y}(Z)$ & $\varepsilon_{Z}(Z)$ \\
B-axis & $\delta_{X}(B)$ & $\delta_{Y}(B)$ & $\delta_{Z}(B)$ & $\varepsilon_{X}(B)$ & $\varepsilon_{Y}(B)$ & $\varepsilon_{Z}(B)$ \\
$W$-axis & $\delta_{X}(W)$ & $\delta_{Y}(W)$ & $\delta_{Z}(W)$ & $\varepsilon_{X}(W)$ & $\varepsilon_{Y}(W)$ & $\varepsilon_{Z}(W)$ \\
A-axis & $\delta_{X}(A)$ & $\delta_{Y}(A)$ & $\delta_{Z}(A)$ & $\varepsilon_{X}(A)$ & $\varepsilon_{Y}(A)$ & $\varepsilon_{Z}(A)$ \\
Cam Angle & -- & -- & -- & -- & -- & $\Delta \alpha$ \\
Turntable & -- & -- & -- & -- & -- & $\Delta \beta$ \\
Angle & & & & & & \\
Center & $\Delta a$ & -- & -- & -- & -- & -- \\
Distance & $\delta_{X}(S)$ & $\delta_{Y}(S)$ & $\delta_{Z}(S)$ & $\varepsilon_{X}(S)$ & $\varepsilon_{Y}(S)$ & $\varepsilon_{Z}(S)$ \\
Turntable
\end{tabular}

Table2. Representation method of transformationmatrix

\begin{tabular}{cccccccc}
\hline \multirow{2}{*}{$\begin{array}{c}\text { Coordinate } \\
\text { System }\end{array}$} & \multicolumn{3}{c}{$\begin{array}{c}\text { Linear } \\
\text { Displacement }\end{array}$} & & \multicolumn{3}{c}{$\begin{array}{c}\text { Angular } \\
\text { isplacement }\end{array}$} \\
\cline { 2 - 4 } \cline { 6 - 8 } Spindle & $t_{T X}$ & $t_{T Y}$ & $t_{T Z}$ & & $r_{R X}$ & $r_{R Y}$ & $r_{R Z}$ \\
B-axis & $t_{B X}$ & $t_{B Y}$ & $t_{B Z}$ & & $r_{B X}$ & $r_{B Y}$ & $r_{B Z}$ \\
A-axis & $t_{A X}$ & $t_{A Y}$ & $t_{A Z}$ & & $r_{A X}$ & $r_{A Y}$ & $r_{A Z}$ \\
$W$-axis & $t_{W X}$ & $t_{W Y}$ & $t_{W Z}$ & & $r_{W X}$ & $r_{W Y}$ & $r_{W Z}$ \\
$Z$-axis & $t_{Z X}$ & $t_{Z Y}$ & $t_{Z Z}$ & & $r_{Z X}$ & $r_{Z Y}$ & $r_{Z Z}$ \\
Cam Angle & -- & -- & -- & & -- & -- & $r_{\alpha}$
\end{tabular}

\begin{tabular}{ccccccc} 
Turntable & - & -- & -- & -- & -- & $r_{\beta}$ \\
Angle & -- & - & & & & \\
Center & & & $t_{a}$ & -- & -- & -- \\
Distance & -- & -- & & & & \\
\hline
\end{tabular}

When deriving the globoidal indexing cam profile equation, there are three rectangular coordinate systems are established, as shown in Fig.2. The coordinate system $O_{0} X_{0} Y_{0} Z_{0}$ is a fixed coordinate system, its origin $O_{0}$ is the intersection point between the center axis of cutter and the swinging centerline of the workpiece, that is, the intersection point of the center axis of the axis $Z$ and the axis $B$. The $O_{0} Z_{0}$ axis overlaps with the centreline of cutter, that is, overlapping withaxis $Z$. The $O_{0} Y_{0}$ axis overlaps with the swinging centerline of the workpiece, that is, overlapping with the centerline when axis Bmove. The coordinate system $O_{1} X_{1} Y_{1} Z_{1}$ oscillates around the axis $B$ and the $O_{1} Z_{1}$ axis overlaps with the workpiece rotation centreline, that is, it overlaps with the centerline whenaxis $A$ move; The $O_{1} X_{1}$ axis overlaps with the line $O_{1} O_{0}$, that is, parallel with the axis $W$. And the coordinate system is fixed with workpiece. Making the coordinate system $O_{1} X_{I} Y_{1} Z_{I}$ rotates around the $O_{1} Z_{1}$ axis, the rotation angle is equal to the angular Displacement of axis $A$ movement, then we can get the coordinate system $O X Y Z$. The point $P$ on the centerline of cutter is a random point on the theoretical profile after processing. The theoretical profile of globoidal indexing cam is generated by using point $P$ and through the generating method. In Fig.2, $b$ is the distance between the point $P$ and the point $O_{0}, d$ isthe diameter of cutter, $a$ is the distance between theaxis $A$ rotation center line and the axis $B$ swing centerline of machine tool, that is the distance between cam rotation center axis line and the cam swing center axis line, which is called center distance. The relation between $\alpha$ and $\beta$ is determined by the motion law of a pre-designed globoidal indexing cam mechanism, which is a known condition.

According to the coordinate system established in Fig.2 and the feature transformation matrix based on multi-body theory, the position vector $p_{0}$ of point $P$ in coordinate system $O_{0} X_{0} Y_{0} Z_{0}$ is as follows:

$$
p_{0}=\left(\begin{array}{c}
x_{0} \\
y_{0} \\
z_{0}
\end{array}\right)=\left(t_{B X} t_{B Y} t_{B Z} r_{B X} r_{B Y} r_{B Z}\right)\left(t_{Z X} t_{Z Y} t_{Z Z} r_{Z X} r_{Z Y} r_{Z Z}\right)\left(t_{T X} t_{T Y} t_{T Z} r_{T X} r_{T Y} r_{T Z}\right) t_{b}
$$

The position vector $p_{l}$ of point $P$ in coordinate system $O_{I} X_{I} Y_{1} Z_{1}$ is as follows:

$$
p_{1}=\left(\begin{array}{c}
x_{1} \\
y_{1} \\
z_{1}
\end{array}\right)=\left(t_{W X} t_{W Y} t_{W Z} r_{W X} r_{W Y} r_{W Z}\right) r_{\beta} p_{0}+t_{a}
$$

The position vector $p$ of point $P$ in the coordinate system $O X Y Z$ is as follows:

$$
p=\left(\begin{array}{l}
x \\
y \\
z
\end{array}\right)=t_{\alpha} p_{1}
$$

The unit normal vector of theoretical profile after machining is as follows: 


$$
n=\frac{\frac{\partial p}{\partial b} \times \frac{\partial p}{\partial \alpha}}{\left|\frac{\partial p}{\partial b} \times \frac{\partial p}{\partial \alpha}\right|}
$$

The actual profiles on both sides of the processing are represented by $p_{l}$ and $p_{r}$ respectively, and the solution is as follows:

$$
\begin{aligned}
& p_{l}=p-\frac{d}{2} n \\
& p_{r}=p+\frac{d}{2} n
\end{aligned}
$$

Adding the equation (9), (10) to the equation (11), (12), the error model of globoidal indexing cam profile could be obtained.

Setting all the error value in table 1 as 0 , then the theoretical profile equation of globoidal indexing cam could be obtained.

\section{Measuring Methods of Cam Profile}

\subsection{MeasuringMethod of Equidistant Surface.}

Only when the ruby measuring ball of CMM contacts with the object to be tested, the position information (coordinate) of the ruby-measuring ball will be recorded. Therefore, the distance between the coordinate and the contact point is the radius $\mathrm{R}$ of ruby measuring ball; All the points on the object to be tested are recorded by CMM, and all the distance from these points to the object under test are the radius $\mathrm{R}$ of ruby measuring ball, when we fit these points into a complete surface, the equidistant model of the object to be tested is obtained, and the distance between the equidistant model and the object to be tested is R. As shown in Fig.3.

\subsection{Modeling Method of Equidistant Surface.}

According to the principle of Equidistant surface, making the point on the theoretical profile deviate along its normal direction, and keeping the deviating distance is equal to the radius of cutter, so that the practical profile can be obtained.

The globoidal cam mechanism is composed of globoidal cam and follower (roller). The motion law of globoidal cam and follower is modified sine motion law, according to the profile equation given in design and application innovation8 of intermittent motion mechanism, the equidistant model of the contact point between cam and follower extending along its normal vector direction is equivalent to the globoidal cam profile equation by reducing the radius of the follower, as shown in Fig.4.

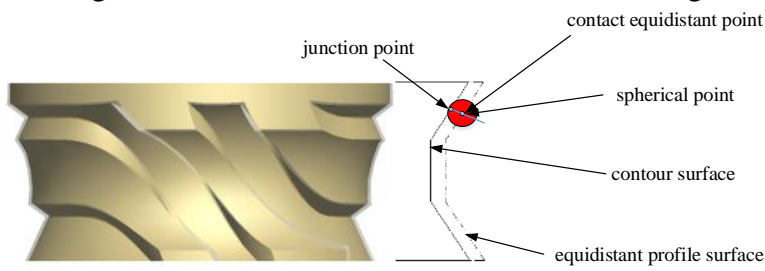

Figure3. Equidistant profile model theory

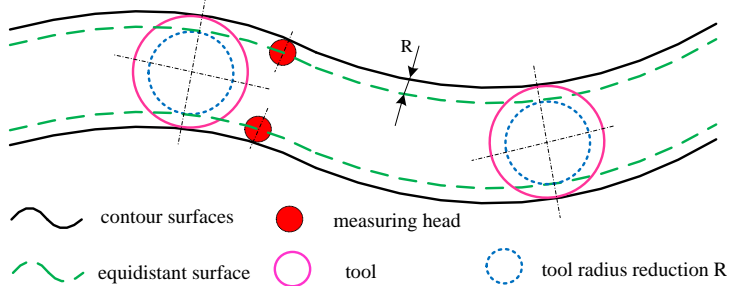

Figure4. Relationship of equidistant model and cutter radius

Taking the globoidal cam as the starting pointand then modifying its theoretical model so as to construct the equidistant model. The measuring result of the CMM is the spherical center coordinate of the measuring ball, and the distance between the contact point and the spherical center is the radius $\mathrm{R}$ of the measuring ball. According to the principle of equidistant surface, making every point $\mathrm{P}$ of the globoidal cam deviate along its normal vector direction $n$, and deviating the fixed distance $\mathrm{R}$, then we can get the corresponding equidistant points of $P$, that is $P_{l}$ ' and $P_{r}$,', which are used as the theoretical equidistant profile model for the coordinate measuring.

$$
\begin{aligned}
& \boldsymbol{P}_{l}^{\prime}=\boldsymbol{P}-\left(\frac{d}{2}-R\right) n \\
& \boldsymbol{P}_{r}^{\prime}=\boldsymbol{P}+\left(\frac{d}{2}-R\right) n
\end{aligned}
$$

\subsection{Analysis of Measuring Principle.}

In order to improve the measuring speed and evaluate the line profile tolerance, the method of measuring the characteristic line of the equidistant surface model is put forward, as shown in Fig.5.

No matter where the contact point of CMM measuring ball and globoidal cam is, only keeping the spherical center of measuring ball moves in the specific axial section, so the trajectory of spherical center must in this axial section, the ideal trajectory of the spherical center is the intersection line of the theoretical equidistant surface and the axial section, thus we can use the characteristic line of equidistant model axial section as the evidence of evaluating the line profile of globoidal cam, and there is no need to compensate the probe radius. In the actual measurement, it is only necessary to make the probe of CMM move along the direction of axial section and just making sure the movement of the spherical center in corresponding axis section of this characteristic line. This measuring method could improve the measuring speed through unchanging the angle of the probe.

The axial section equation at $\alpha$ angle is as follows:

$$
y / x=\tan (\alpha)
$$

According to the definition of the equidistant profile of globoidal cam and the characteristic line of the axial section, we can form an equation sets through combining the formula(13) or formula(14) of equidistant profile of globoidal cam respectively with axial equation(15) to form 
a simultaneous equation, and the result is the characteristic line of equidistant profile model.

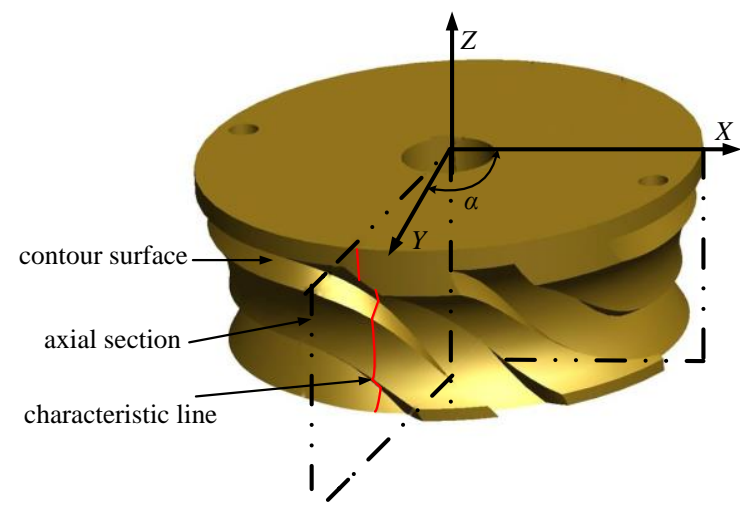

Figure5. Characteristic line diagram

\subsection{Construction of Measurement System.}

Using the high-precisionCMM to build precision turntable so as to form the four axis measurement system, achieving rapid measurement of tolerance about globoidal cam profile, the overall structure of the measurement system is shown in Fig.6. The measurement system is composed of CMM, precision turntable, motor controller, driver and digital display. The digital display shows angle information of turntable in real time, processing the information and the measuring results through computer so as to improve the measuring speed and accuracy.

\section{Measurement Experiment and Analysis}

\subsection{Composition of Measurement System.}

The whole set of device of tolerance detection system about globoidal indexing cam profile as shown in Fig.7, the specific experimental conditions includes: CMM, model NHC-Y564, measurement range is $X$ axis $-500 \mathrm{~mm}$, $\mathrm{Y}$ axis $-600 \mathrm{~mm}, \mathrm{Z}$ axis $-400 \mathrm{~mm}$; precision turntable system includes: the turntable and the controller, circular grating angle measurement system and the positioning module; the work piece be measured, TC40 type of globoidal indexing cam, size: thickness of $97 \mathrm{~mm}$, maximum diameter of $265 \mathrm{~mm}$.

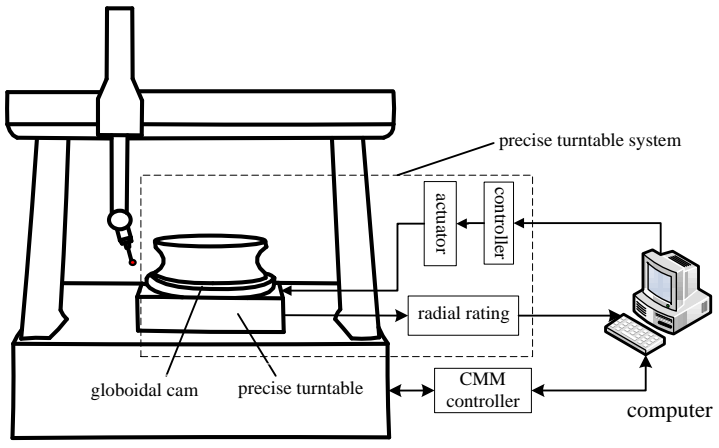

Figure6. Structural diagram of measuring device

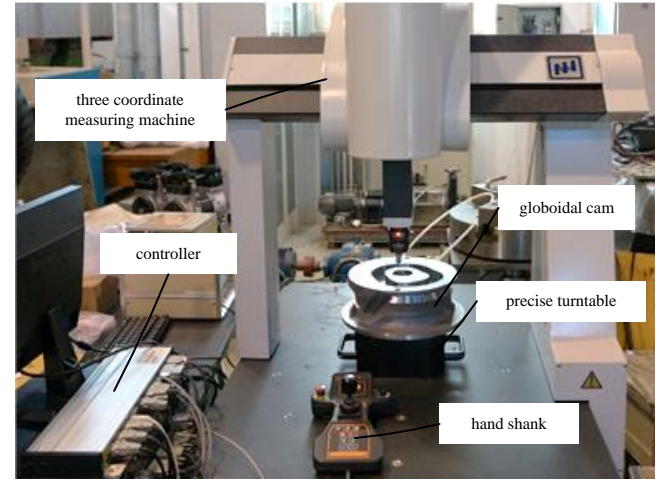

Figure7. Set-up diagram of measurement system

\subsection{Process of Measurement Experiment.}

Taking the globoidal cam profile equidistant model as the theoretical model, based on the manufacturing datum of the globoidal cam, establishing the datum coordinate system, which makes the globoidal cam and its theoretical model in the same coordinate system. During the measurement process, after a characteristic line has been measured, controlling the precision turntable rotates to the next characteristic line which is going to be measured. Combining the measuring method of the globoidal cam equidistant model with the above referred device, the measuring process is as follows.

Firstly, establish a reference coordinate system. Secondly, found a measurement coordinate system. Thirdly, measuring the feature points while adjusting the turntable angle. Export data of feature points at the end of measurement.

Byselecting different measuring angles, the equidistant characteristic point of the corresponding angle can be measured, and comparing it with the theoretical characteristic line under the same angle, calculating to get the line profile tolerance of the globoidal cam equidistant model .

\subsection{Analysis of Measurement Results.}

Taking this paper takes the TC40 globoidal cam as an example, the data point coordinates obtained from the CMM are shown in Table3:

The data in table 1 are the characteristic point coordinates of the globoidal cam intermittent section and PT1-PT8 is the characteristic line of the upper part in Fig.5; PT9-PT16 is the characteristic line of the lower part of Fig.5.

Calculating the distance of actual measuring characteristic point Pt by using Matlab according to the method described in 3, the results are shown in Fig.8. 


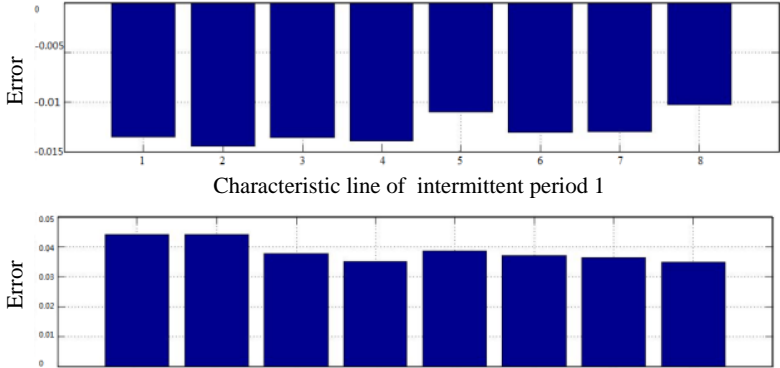

Characteristic line of intermittent period 2

Figure8. Line profile tolerance

The maximum value Dtmax from the characteristic point $\mathrm{Pt}$ to the theoretical characteristic line $\mathrm{Si}$ is 0.043 $\mathrm{mm}$ and the minimum value of Dtmin is $-0.014 \mathrm{~mm}$.

$$
\Delta L=D_{\text {tmax }}-D_{\text {tmin }}=0.043-(-0.01)=0.057 \mathrm{~mm}
$$

Therefore, the line profile tolerance of the characteristic line under the cam angle is $\Delta \mathrm{L}=0.057 \mathrm{~mm}$.

Table3. Coordinates of characteristic points on dwell segment

\begin{tabular}{cccc}
\hline Point & Coordinate & $\begin{array}{c}\text { Theory } \\
(\mathrm{mm})\end{array}$ & $\begin{array}{c}\text { Practical } \\
(\mathrm{mm})\end{array}$ \\
PT1 & $\mathrm{X}$ & 122.2390 & 122.2735 \\
& $\mathrm{Z}$ & -18.5961 & -18.6978 \\
PT2 & $\mathrm{X}$ & 122.9901 & 123.0258 \\
& $\mathrm{Z}$ & -19.8971 & -20.0028 \\
PT3 & $\mathrm{X}$ & 123.7412 & 123.7742 \\
& $\mathrm{Z}$ & -21.1980 & -21.2973 \\
PT4 & $\mathrm{X}$ & 124.4923 & 124.5281 \\
& $\mathrm{Z}$ & -22.4989 & -22.6038 \\
PT5 & $\mathrm{X}$ & 125.2433 & 125.2835 \\
& $\mathrm{Z}$ & -23.7999 & -23.9063 \\
PT6 & $\mathrm{X}$ & 125.9944 & 126.0328 \\
& $\mathrm{Z}$ & -25.1008 & -25.2083 \\
PT7 & $\mathrm{X}$ & 126.7455 & 126.7817 \\
& $\mathrm{Z}$ & -26.4017 & -26.5053 \\
PT8 & $\mathrm{X}$ & 127.4966 & 127.5366 \\
& $\mathrm{Z}$ & -27.7026 & -27.8074 \\
PT9 & $\mathrm{X}$ & 107.5998 & 107.6338 \\
& $\mathrm{Z}$ & -73.2422 & -73.3760 \\
PT10 & $\mathrm{X}$ & 108.9003 & 108.9354 \\
& $\mathrm{Z}$ & -72.4913 & -72.6245 \\
PT11 & $\mathrm{X}$ & 110.2007 & 110.2345 \\
PT13 & $\mathrm{Z}$ & -71.7405 & -71.8670 \\
& $\mathrm{X}$ & 111.5012 & 111.5381 \\
& $\mathrm{Z}$ & -70.9897 & -71.1115 \\
& $\mathrm{X}$ & 112.8017 & 112.8387 \\
& $\mathrm{Z}$ & -70.2389 & -70.3646 \\
& $\mathrm{X}$ & 114.1021 & 114.1348 \\
& $\mathrm{Z}$ & -69.4880 & -69.6146 \\
& $\mathrm{Z}$ & 115.4026 & 115.4384 \\
& & -68.7372 & -68.8611 \\
& & 116.7031 & 116.7395 \\
& -67.9864 & -68.1081 \\
\hline
\end{tabular}

The measuring precision of the globoidal cam intermittent section is pretty high, the tolerance between each point is rather small, and it proves that the measuring method of the equidistant model is suitable for the intermittent section. The reason why there is slightly larger deviation between $\mathrm{X}$ coordinate and the $\mathrm{Z}$ coordinate is that the profile cutting amount is pretty large when the cam is processed, and there is a slight gap between the globoidal cam and the roller, causing the phenomenon of 'jitter'.

Taking TC40 type globoidal cam as an example, measuring the eight characteristic lines (4 in intermittent section and 4 in indexing section). The calculation results of line profile tolerance of each characteristic line are shown in Table4.

Table4. Calculation results of line profile tolerance

\begin{tabular}{ccc}
\hline \multicolumn{3}{c}{ line profile tolerance in intermittent section } \\
\hline characteristic line & $D_{\text {tmax }}$ & $D_{\text {tmin }}$ \\
L1 & 0.043 & -0.014 \\
L2 & 0.038 & -0.021 \\
L3 & 0.041 & -0.016 \\
L4 & 0.035 & -0.019 \\
L5 & 0.213 & -0.339 \\
L6 & 0.014 & -0.552 \\
L7 & 0.221 & -0.281 \\
L8 & 0.436 & -0.267 \\
\hline
\end{tabular}

When the globoidal cam indexing mechanism is working in the intermittent section, the driven turnplate will stop from moving, and the exchange cutter manipulator will carry out the operation of grasping cutter and setting cutter. In order to prevent the tools from falling, the movement should be stable. The pretty high machining accuracy of globoidal cam profile can increase the density of measurement and improve the accuracy of tolerance detection. When working in the indexing section, the globoidal cam drives the driven turnplate to do rotary movement, and the driven turnplate drives the exchange cutter manipulator to finish the cutter exchange operation, in this process, we need to ensure the rotation precision and stability, so that make the manipulator working precisely in place. In addition, the phenomenon of vibration and entrapment should be avoided. In the indexing section, the change of curvature is large, the processing is difficult, and the contact measurement of cam profile brings about pretty big difficulty, the profile tolerance is also relatively large, so the measurement density can be reduced appropriately.

\section{Summary}

This paper researches on the influence caused by error of globoidal cam special machine tool on the error of globoidal cam profile, the machining error of special machine tool and coordinate transformation matrix is analyzed by using multi-body system theory, at the same time, the error model of the globoidal indexing cam profile is established, which lays certain foundation for the integration of design, processing and measurement to globoidal cam mechanism. The main conclusions are as follows:

1) According to the structure of globoidal cam special machine tool, establishing the machine tool error 
transformation matrix based on multi-body system theory, and the mathematical model of globoidal cam profile error is established according to the generating machining theory of globoidal cam.

2) The establishment of mathematical model of globoidal cam profile machining error lays the theoretical basis for precision design and error distribution of special machine tool for globoidal cam profile, the quality detection and error evaluation of globoidal cam profile, and the tracing for globoidal cam machining error.

3) According to the method of generalizing machining and the equidistant model principle, establishing the theoretical profile equation of globoidal cam, the equidistant model equation and the axial sections characteristic line equation, which are used to evaluate the tolerance of the globoidal cam profile.

Putting forward the tolerance evaluation method of profile of the globoidal cam equidistant model, and then verifying it through experiment, experimental results are analyzed and the influence caused by globoidal cam profile tolerance on ATC transmission is discussed. This establishes the theoretical foundation for further separation and tracing of machining tolerance, establishment of the evaluation system and measuring standard about globoidal cam profile tolerance, and achieving the integration of design, machining and measurement.

\section{Acknowledgments}

This research was funded by the National Science and Technology Major Project, China, under contract 2013ZX04008-021. The authors would like to acknowledge the support and contribution from the Beijing No. 1 Machine Tool Plant for their help with manufacturing and be grateful to the Key Lab of Mechanical Engineering, Beijing University of Technology, China.

\section{References}

1. Han Yuemei. Research progress of automatic tool changer in machining center[J]. Equipment Manufacturing Technology, 2010, (5): 128-129.

2. Zhang Gaofeng, Yang Shiping, Chen Huazhang etc. Research and prospect of globoidal indexing CAM mechanism [J]. Mechanical Drive, 2003, 27(3): 1-5.

3. He Yi, Liu Yansong, Wang Tao etc. The review and prospect of arc indexing CAM mechanism research[J]. Light Industrial Machinery, 2003, (4): 7-10.

4. BU Fan-hua. Study on machining technology of globoidal cam employed in automatic tool changer (ATC) of machine center [D]. Beijing:College of Mechanical Engineering and Applied Electronics Technology, Beijing University of Technology, 2012.
5. W H Wang, C H Tseng, C B Tsay. Surface Contact Analysis for a Spatial Cam Mechanism[J]. Transaction of the ASME Journal of Mechanical Design, 1997, 119: 169-177.

6. H Y Cheng. Optimum Tolerance for Globoidal Cam Mechanisms [J]. JSME International Journal, Series C. 2002, 45(2): 519-526.

7. Yin M F, Lyu C Y, Zhao Z H. The theoretical study of CAM processing theory and the calculation method of the influence of CAM body deviation error on the profile error are studied[J]. Journal of shandong university of technology (natural science edition), 2001, 15(3):18-21.

8. Ji S T, Zhang Y M, Zhao J. The influence of center distance error of machining center of globoidalcam on convex contour error[J]. Journal of Beijing University of Technology, 2014, 40(6): 825-830.

9. Zhang Y M, Ji S T. Zhao J. Tolerance Analysis and Allocation of Special Machine Tool for Manufacturing Globoidal Cams [J]. International Journal of Advanced Manufacturing Technology, 2016,87:1597-1607.

10. Bu F H, Zhang Y M,Shang D G. Study on Machining Error of Globoidal Cam Profile Resulting from Rotational Deviation of Location of Part in Machining[C]].Advanced Materials Research 2012, 452-453:211-218.

11. WANG Hai-yan, ZHAO Ru-jia, LIU Chang-qi. Precision measurement and error evaluation of globoidal cam [J].Measurement Technique, 1999, 6:9-12.

12. WANG Xiao-fei, TANG Lin. Measurement theory and implement of globoidal indexing cam using $\mathrm{cmm}[\mathrm{J}]$. Journal of Tianjin University, 2011,44(11): 1024-1028.

13. P D Lin, J F Hsieh. Dimension inspection of spatial cams by cnc coordinate measuring machines[J]. Transaction of the ASME Journal of Manufacturing Science and Engineering, 2000, 122:149-157.

14. HU Bing-chen, KONG Qing-yi, XU Lian-tang. The discussion of precision index system of globoidal cam $[\mathrm{J}]$. Transactions of the Chinese Society for Agricultural Machinery, 1994,25:13-16.

15. SONG Li-juan. Measurement of three-coordinate machine for globoidal indexing CAM[D].Dalian: College of Mechanical Engineering, Dalian University of Technology, 2005.

16. LIN Xiao-jun, WANG Zeng-qiang, SHAN Chen-wei. Method of probe radius compensation for free 
surface measurement by CMM [J]. Aeronautical Manufacturing Technology, 2011, 10:75-78. 